\title{
Dermoscopic and reflectance confocal microscopic presentation of relapsing eccrine porocarcinoma
}

\author{
Rita Pinheiro, MD, ${ }^{\mathrm{a}}$ André Oliveira, MD, ${ }^{\mathrm{b}, \mathrm{c}}$ and Pedro Mendes-Bastos, $\mathrm{MD}^{\mathrm{d}}$ \\ Lisboa, Portugal
}

\section{CLINICAL PRESENTATION}

A 63-year-old woman presented with an exophytic polypoid tumor on her left arm, adjacent to a previous scar. An eccrine porocarcinoma (EPC) had been excised 10 months before presentation (Fig 1, $A$ ).

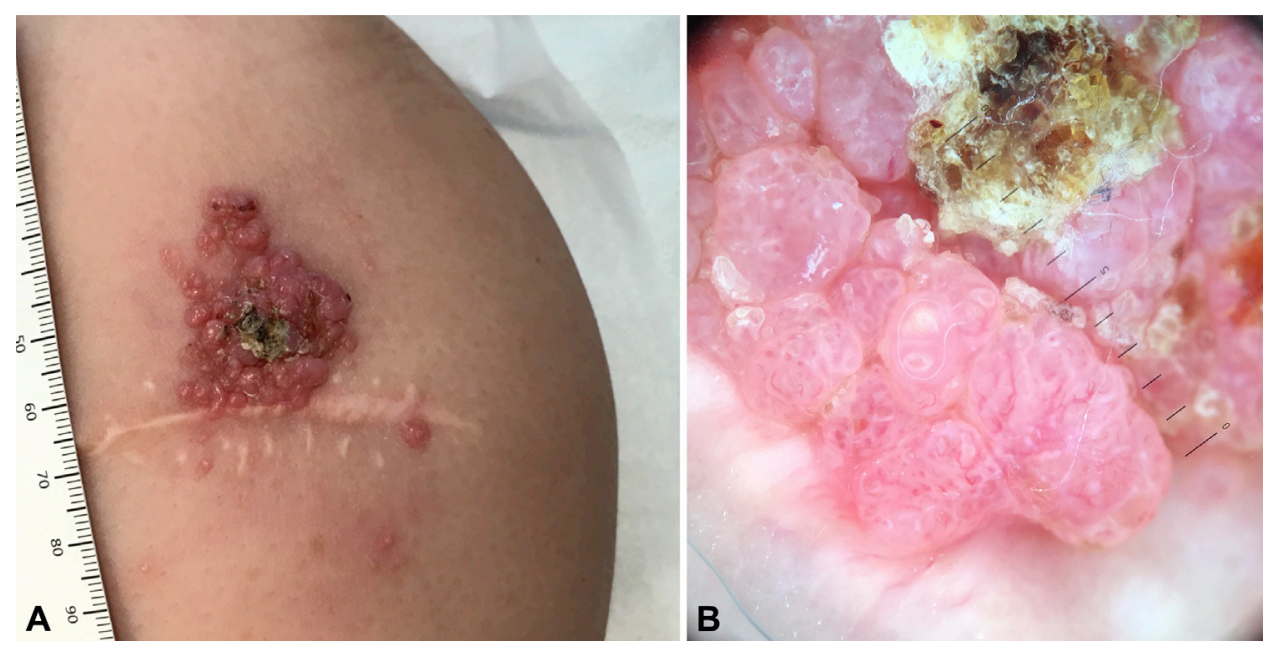

Fig 1. Clinical and dermoscopic presentations of relapsing eccrine porocarcinoma. A, A poorly defined exophytic tumor with multiple clustered pink papules on the left arm, $3 \mathrm{~cm}$ in maximum diameter, adjacent to a previous scar; few satellite papules are seen. B, Dermoscopy reveals a lobular arrangement of multiple polymorphic vessels (ie, linear-irregular, arboriform, and coiled) surrounded by peripheral white-pink halos; ulceration is also seen.

\section{DERMOSCOPIC APPEARANCE}

Lobular aggregates were seen with a diffuse arrangement of focused and unfocused polymorphous (ie, linear-irregular, arboriform, and coiled) vessels within, surrounded by a white-pink halo; ulceration was also noted (Fig 1,B).

\section{CONFOCAL MICROSCOPY APPEARANCE}

Reflectance confocal microscopic (RCM) features are shown in Fig 2.

\footnotetext{
From the Department of Dermatology, ${ }^{a}$ Centro Hospitalar de Lisboa Central; Department of Dermatology, ${ }^{\mathrm{b}}$ Hospital de Santa Maria; Centro Académico Médico de Lisboa, ${ }^{\mathrm{C}}$ Universidade de Lisboa; and the Dermatology Centre, ${ }^{\mathrm{d}}$ Hospital CUF Descobertas.

Publication of this article was supported by 3 Gen Inc.

Funding sources: None.

Conflicts of interest: None declared.

Correspondence to: André Oliveira, MD, Department of Dermatology, Hospital de Santa Maria, Avenida Professor
}

\footnotetext{
Egas Moniz, Lisboa 1649-035, Portugal. E-mail: andre.oliveira@ sapo.pt.

J Am Acad Dermatol 2017;76:S73-5.

0190-9622/\$36.00

(c) 2016 by the American Academy of Dermatology, Inc.

http://dx.doi.org/10.1016/j.jaad.2016.08.020
} 

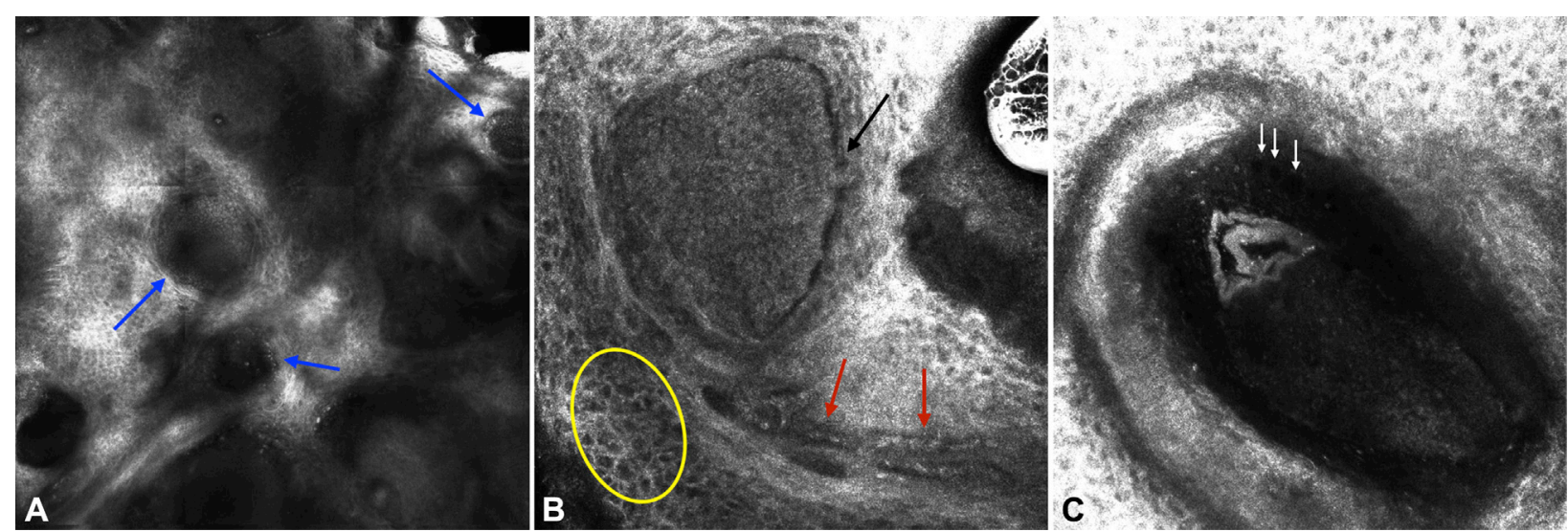

Fig 2. Reflectance confocal microscopy appearance of relapsing eccrine porocarcinoma. $\mathbf{A}$, Mosaic image $(1.5 \mathrm{~mm} \times 1.5 \mathrm{~mm})$ at the epidermal level enables the observation of round refractile tumoral islands (blue arrows) surrounded by dark stroma; multiple atypical, nonpalisading, small, cuboidal cells with dark nuclei and bright cytoplasm are seen within. $\mathbf{B}$, Detail of a tumoral island (black arrow) surrounded by elongated and tortuous canalicular vessels (red arrows) and typical (yellow circle) honeycomb pattern (basic image, $0.5 \mathrm{~mm} \times 0.5 \mathrm{~mm})$. C, Roundish dark structures corresponding to areas of ductal differentiation (white arrows) within a nest are also seen. Dark stroma surrounding clustered atypical bright cells is featured in detail (basic image, $0.5 \mathrm{~mm} \times 0.5 \mathrm{~mm}$ ).

\section{HISTOLOGIC DIAGNOSIS}

The clinical, dermoscopic, and confocal microscopic correlation suggested the diagnosis of relapsing EPC, which was supported after obtaining a punch biopsy specimen. The histopathologic examination revealed multiple nests and cords of neoplastic poroid cells separated by confluent sheets of intervening stroma, both on the reticular and papillary dermis (Fig 3, A). Scattered areas of incipient tubular differentiation stained positive for carcinoembryonic antigen (Fig 3, B).
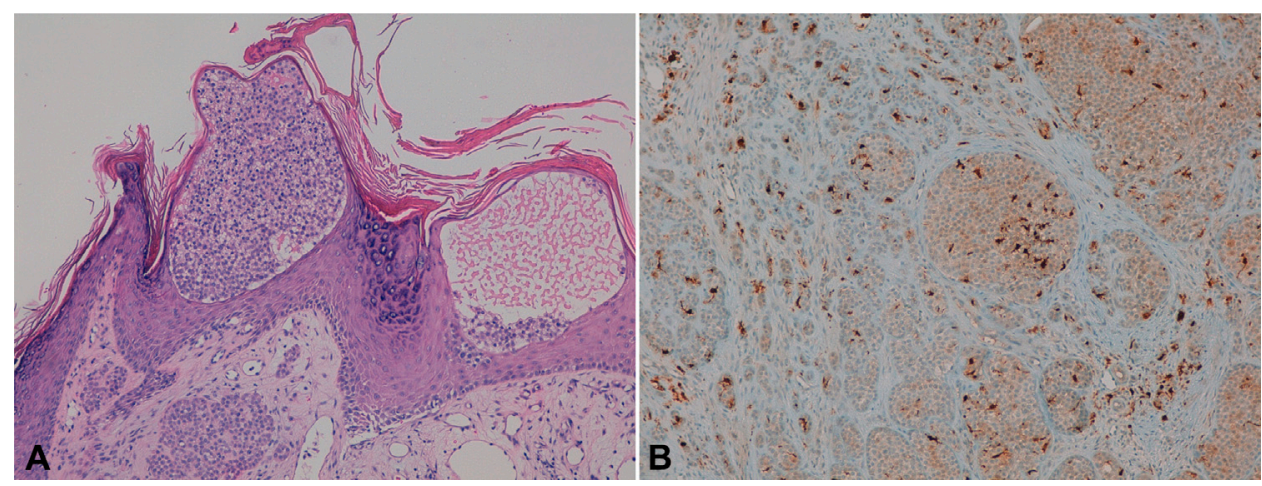

Fig 3. Histopathologic examination of relapsing eccrine porocarcinoma. A, Ulcerated tumor with an irregular infiltrating border on the reticular dermis. Multiple intraepidermal and dermal nests and cords of small poroid tumoral cells are seen. (Hematoxylin-eosin stain; original magnification, $\times 200$.) B, Incipient tubular differentiation is highlighted by positive carcinoembryonic antigen staining. 


\section{KEY MESSAGE}

EPC is a rare malignant sweat gland tumor. ${ }^{1}$ We showed that aggregates of poroid cells were seen clustered in nests, surrounded by dark stroma and canalicular vessels on RCM, also correlating to white-pink halos observed on dermoscopy. Round dark structures within the nests reflected its ductal differentiation. Therefore, dermoscopy and RCM can provide valuable noninvasive clues for the diagnosis and follow-up of patients with EPC. ${ }^{2}$ Our case highlights not only the aggressive behavior of relapsing EPC but also the novel cytomorphologic features under RCM. These findings should be further explored for possible presurgical assessment of EPC considering its high local recurrence rate.

\section{REFERENCES}

1. Sgouros D, Piana S, Argenziano G, et al. Clinical, dermoscopic and histopathological features of eccrine poroid neoplasms. Dermatology. 2013;227:175-179.

2. Brugués A, Gamboa M, Alós L, Carrera C, Malvehy J, Puig S. The challenging diagnosis of eccrine poromas. J Am Acad Dermatol. 2016;74:e113-e115. 